\title{
Acoplanarity of a Lepton Pair to Probe the Electromagnetic Property of Quark Matter
}

\author{
Spencer Klein, ${ }^{1}$ A. H. Mueller, ${ }^{2}$ Bo-Wen Xiao, ${ }^{3,4}$ and Feng Yuan ${ }^{1}$ \\ ${ }^{1}$ Nuclear Science Division, Lawrence Berkeley National Laboratory, Berkeley, California 94720, USA \\ ${ }^{2}$ Department of Physics, Columbia University, New York, New York 10027, USA \\ ${ }^{3}$ Key Laboratory of Quark and Lepton Physics (MOE) and Institute of Particle Physics, \\ Central China Normal University, Wuhan 430079, China \\ ${ }^{4}$ Centre de Physique Théorique, École Polytechnique, CNRS, Université Paris-Saclay, \\ Route de Saclay, 91128 Palaiseau, France
}

(Received 28 November 2018; revised manuscript received 4 March 2019; published 3 April 2019)

\begin{abstract}
We investigate the $P_{T}$-broadening effects in dilepton production through photon-photon scattering in heavy ion collisions. The QED multiple interaction effect with the medium is found to be consistent with the recent observation of a low transverse momentum lepton pair from the ATLAS Collaboration at the LHC. We further comment on the magnetic broadening effect and point out a number of ways to disentangle these two mechanisms. In particular, the rapidity dependence of the $P_{T}$-broadening effect provides a unique probe to the magnetic effect.
\end{abstract}

DOI: 10.1103/PhysRevLett.122.132301

Introduction.- -Jet quenching is considered one of the major discoveries in relativistic heavy ion experiments from RHIC at Brookhaven National Laboratory and the LHC at CERN [1-4]. This phenomenon has been well formulated in QCD [5-15], which indicates that the energy loss and medium $P_{T}$-broadening effects are closely related. The parameter $\hat{q}$ has been extracted from various experimental data, see, e.g., Refs. [16-20]. Meanwhile, in addition to the vacuum Sudakov $P_{T}$ broadening, $\hat{q} L$ describes the typical transverse momentum squared that a parton acquires after traversing the nuclear medium of length $L$. Generally speaking, the sum of the aforementioned vacuum and medium effects gives the total amount of $P_{T}$ broadening, which can be measured in experiments. In the last few years, there has been significant progress in understanding the $P_{T}$-broadening effects in dijet, photonjet, and hadron-jet productions in heavy ion collisions [21-28]. At the LHC, the dominant broadening effect comes from the vacuum Sudakov contribution [21] for the typical dijet kinematics $[3,4]$. On the other hand, the medium broadening effect is comparable to the Sudakov effects at RHIC, and the STAR measurements have demonstrated the signal of the medium $P_{T}$-broadening effects in hadron-jet correlation [23,27]. Future measurements at both the LHC and RHIC should provide further information on the jet $P_{T}$-broadening physics.

Published by the American Physical Society under the terms of the Creative Commons Attribution 4.0 International license. Further distribution of this work must maintain attribution to the author(s) and the published article's title, journal citation, and DOI. Funded by SCOAP ${ }^{3}$.
More recently, both the ATLAS [29] Collaboration at the LHC and the STAR [30] Collaboration at RHIC have found a new place to look for $P_{T}$-broadening effects-in the final states of dileptons that have been produced by the purely electromagnetic two-photon reaction: $\gamma \gamma \rightarrow \ell^{+} \ell^{-}$. This reaction has been extensively studied in ultraperipheral collisions (UPCs), where it is generally well described by lowest order quantum electrodynamics [31-35]. The lepton pairs have a very small pair $P_{T}$ (tens of $\mathrm{MeV}$ ), so the leptons are nearly back to back. That is to say that the path of either lepton is almost coplanar with respect to the plane containing the beam line and the path of the other lepton. However, ATLAS and STAR observed significant $P_{T}$-broadening effects in dileptons from the reaction $\gamma \gamma \rightarrow \ell^{+} \ell^{-}$, in peripheral and (for ATLAS) central collisions. This broadening hardens the STAR $p_{\perp}^{2}$ spectrum, and allows ATLAS to see a significant loss of coplanarity in moving from UPCs to central collisions. ATLAS also observes a small (order $1 \%$ of the events) tail of events with high acoplanarity, even in UPCs [36].

In this Letter, we study the mechanisms that can lead to this broadening. We extend our previous studies on the dijet azimuthal correlation to the dilepton correlation and focus on two main areas. One is the QED Sudakov effect, where we show that the theory prediction for the UPC events agrees very well with data from ATLAS [36]. Second, we investigate the medium effects, including the QED multiple interaction effects similar to the medium $P_{T}$ broadening of the QCD jet and the magnetic effects [30]. We also discuss how to disentangle these two mechanisms.

The comparison of the $P_{T}$-broadening effects in QCD and QED is of crucial importance to understand the medium property in heavy ion collisions. The lepton's 
$P_{T}$-broadening effects are sensitive to the electromagnetic property of the quark-gluon plasma, whereas the jet $P_{T}$-broadening effects depend on the strong interaction property. The experimental and theoretical investigations of both phenomena will deepen our understanding of the hot medium created in these collisions. The clear indication of lepton $P_{T}$-broadening effects from ATLAS and STAR $[29,30]$ should stimulate further study on dijet azimuthal correlations in heavy ion collisions.

The rest of the Letter is organized as follows. We first study the azimuthal angular correlation for dileptons in UPCs in Sec. II. Then, we investigate the medium effects, including the QED multiple scattering effects and the magnetic effects in Secs. III and IV, respectively. Finally, Sec. V summarizes the paper.

Lepton pair production in ultraperipheral heavy ion collisions. - The leading order production of lepton pairs comes from photon-photon scattering, see, Fig. 1(a). The outgoing leptons have momenta $p_{1}$ and $p_{2}$, individual transverse momenta $p_{1 \perp}$ and $p_{2 \perp}$, and rapidities $y_{1}$ and $y_{2}$, respectively. The leptons are produced dominantly back to back in the transverse plane, i.e., $\left|\vec{p}_{\perp}\right|=\left|\vec{p}_{1 \perp}+\vec{p}_{2 \perp}\right| \ll$ $\left|p_{1 \perp}\right| \sim\left|p_{2 \perp}\right|$. The incoming photons have the following momenta: $k_{1}=P_{\perp} / \sqrt{s}\left(e^{y_{1}}+e^{y_{2}}\right) P_{A}$ and $k_{2}=$ $P_{\perp} / \sqrt{s}\left(e^{-y_{1}}+e^{-y_{2}}\right) P_{B}$, where $P_{\perp}$ represents $\left|p_{1 \perp}\right| \sim$ $\left|p_{2 \perp}\right|$, and the incoming nuclei have per-nucleon momenta $P_{A}$ and $P_{B}$. In the Sudakov resummation formalism, the differential cross section can be written as [37]

$\frac{d \sigma^{A B_{[\gamma]} \rightarrow \mu^{+} \mu^{-}}}{d y_{1} d y_{2} d^{2} p_{1 \perp} d^{2} p_{2 \perp}}=\sigma_{0} \int \frac{d^{2} r_{\perp}}{(2 \pi)^{2}} e^{i p_{\perp} \cdot r_{\perp}} W\left(b_{\perp} ; r_{\perp}\right)$,

where $b_{\perp}$ denotes the centrality at a particular impact parameter of $A A$ collisions, $\sigma_{0}=\left|\overline{\mathcal{M}}^{(0)}\right|^{2} / 16 \pi^{2} Q^{4}$ with $\left|\overline{\mathcal{M}}_{0}\right|^{2}=(4 \pi)^{2} \alpha_{e}^{2} 2\left(t^{2}+u^{2}\right) / t u, Q$ is the invariant mass for the lepton pair, $t$ and $u$ are the usual Mandelstam variables for the $2 \rightarrow 2$ process. In the coordinate space which allows one to conveniently take care of the transverse momentum conservation, $W\left(b_{\perp} ; r_{\perp}\right)$ is the combination of incoming photon fluxes considered in previous studies [38-42] and all order Sudakov resummation (see, e.g., Refs. [21,22,43]),

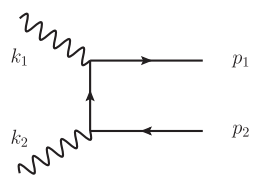

(a)

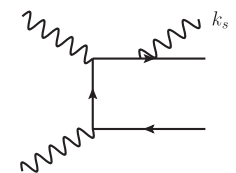

(b)

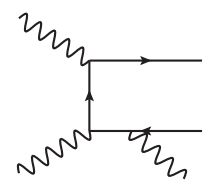

(c)
FIG. 1. The leading order and next-to-leading order QED Feynman diagrams for lepton pair production through $\gamma \gamma$ processes: (a) The leading order diagram (interchange between $k_{1}$ and $k_{2}$ should be included as well); (b) soft photon radiation from the lepton; (c) soft photon radiation from the antilepton. Photon radiation from the lepton propagator is power suppressed.

$$
W\left(b_{\perp} ; r_{\perp}\right)=\mathcal{N}_{\gamma \gamma}\left(b_{\perp} ; r_{\perp}\right) e^{-S_{u}\left(Q, m_{\mu} ; r_{\perp}\right)},
$$

where $S_{u}$ is the Sudakov factor and will be presented below. By setting $S_{u}=0$, one gets back to the results in previous studies [38-41]. The factor $\mathcal{N}_{\gamma \gamma}$ represents the incoming photon flux overlap,

$$
\begin{aligned}
\mathcal{N}_{\gamma \gamma}\left(b_{\perp} ; r_{\perp}\right)= & x_{a} x_{b} \int d^{2} k_{1 \perp} d^{2} k_{2 \perp} e^{i\left(k_{1 \perp}+k_{2 \perp}\right) \cdot r_{\perp}} \\
& \times\left[f_{A}^{\gamma}\left(x_{a}, k_{1 \perp}\right) f_{B}^{\gamma}\left(x_{b}, k_{2 \perp}\right)\right]_{b},
\end{aligned}
$$

where $x_{a}=k_{1} / P_{A}$ and $x_{b}=k_{2} / P_{B}$. To simplify the above expression, we have introduced an impact parameter $b_{\perp}$-dependent photon flux: $\left[f_{A}^{\gamma} f_{B}^{\gamma}\right]_{b}=$ $\int d^{2} b_{1 \perp} d^{2} b_{2 \perp} \Theta\left(b_{\perp}\right) N_{\gamma}\left(b_{1 \perp}, k_{1 \perp}\right) N_{\gamma}\left(b_{2 \perp}, k_{2 \perp}\right)$, where $\Theta(b)$ denotes the impact parameter constraints for a particular centrality with $\vec{b}_{\perp}=\vec{b}_{1 \perp}-\vec{b}_{2 \perp}$, and individual photon flux $N_{\gamma}\left(b_{1 \perp}, k_{1 \perp}\right)$ can be computed separately [38-42]. Here, the interdependence between the impact parameter $b_{i \perp}$ and the photon's transverse momentum contribution $k_{i \perp}$ is ignored, which could introduce additional theoretical uncertainties.

The Sudakov factor $S_{u}$ starts to appear at one-loop order, where soft photon radiations contribute to the dominant logarithms in the kinematics of our interest. The typical Feynman diagrams for the real photon radiation are shown in Figs. 1(b),1(c). Applying the Eikonal approximation, see, e.g., Ref. [43], we obtain

$$
\left.\mathcal{M}^{(1) r}\right|_{\text {soft }} ^{2}=e^{2} \frac{2 p_{1} \cdot p_{2}}{p_{1} \cdot k_{s} p_{2} \cdot k_{s}}\left|\mathcal{M}^{(0)}\right|^{2},
$$

where $\mathcal{M}^{(0)}$ is the leading order Born amplitude and $k_{s}$ is the soft photon momentum. In the small total transverse momentum region $\ell_{\perp}=p_{\perp} \ll P_{\perp}$, we have the following behavior from the above contribution: $\left(\alpha / \pi^{2}\right)\left(1 / \ell_{\perp}^{2}\right) \ln \left(Q^{2} / \ell_{\perp}^{2}+m_{\mu}^{2}\right)$, where $m_{\mu}$ is the lepton mass and $\ell_{\perp}$ is related to $k_{s \perp}$. In order to derive the oneloop result for $S_{u}$, we need to Fourier transform the above expression to the conjugate $r_{\perp}$ space, and add the virtual photon contributions. Because of the lepton mass $m_{\mu}$, the cancellation between the real and virtual diagrams will depend on the relative size of $\mu_{r}=c_{0} / r_{\perp}$ and $m_{\mu}$, where $c_{0}=2 e^{-\gamma_{E}}$ with $\gamma_{E}$ the Euler's constant. In the end, we find at one-loop order [37],

$$
S_{u}= \begin{cases}-\frac{\alpha}{2 \pi} \ln ^{2} \frac{Q^{2}}{\mu_{r}^{2}}, & \mu_{r}>m_{\mu}, \\ -\frac{\alpha}{2 \pi} \ln \frac{Q^{2}}{m_{\mu}^{2}}\left(\ln \frac{Q^{2}}{\mu_{r}^{2}}+\ln \frac{m_{\mu}^{2}}{\mu_{r}^{2}}\right), & \mu_{r}<m_{\mu} .\end{cases}
$$

When the lepton mass is negligible, i.e., $\mu_{r} \gg m_{\mu}$, this leads to the same leading double logarithmic behavior as that in the back-to-back hadron production in $e^{+} e^{-}$ 


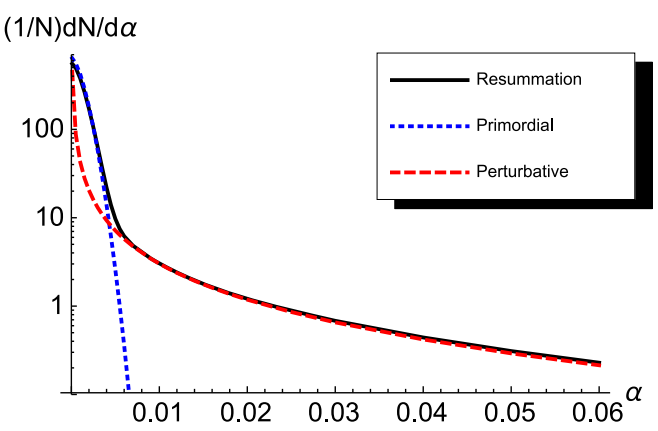

FIG. 2. Acoplanarity distribution for lepton pair production at midrapidity in UPC events at the LHC with typical kinematics cuts: Lepton transverse momentum $P_{\perp}>4 \mathrm{GeV}$ and pair invariant mass from 10 to $100 \mathrm{GeV}$. The total contribution with resummation (solid curve) agrees well with the ATLAS measurement [36].

annihilation studied in Refs. [44-46]. This provides an important cross check for our results.

Combining the above Sudakov result with the incoming photon fluxes contributions in Eq. (2), we can calculate the total transverse momentum distribution. In order to simplify the numeric evaluation, we parametrize the $k_{i \perp}$ dependence for the incoming photon flux as simple Gaussian distributions with a typical width around $40 \mathrm{MeV}$. The Gaussian width is also consistent with a fit to the STARlight $[38,42]$ simulation. In ATLAS, the azimuthal angular correlation of the lepton pair is studied: $\phi_{\perp}=\pi-\left(\phi_{1}-\phi_{2}\right)$, where $\phi_{1}$ and $\phi_{2}$ represent the azimuthal angles for the lepton and the antilepton, respectively. Figure 2 compares different contributions to this correlation as a function of the acoplanarity $\alpha=\left|\phi_{\perp}\right| / \pi$ at the LHC for the lepton pair production at midrapidity with lepton transverse momentum $P_{\perp}>4 \mathrm{GeV}$ and invariant mass $10 \mathrm{GeV}<M_{\mu \mu}<100 \mathrm{GeV}$. The dotted line represents the primordial contribution from the two photon's transverse momenta, the dashed curve stands for the perturbative one soft photon radiation, and the solid curve corresponds to the total contribution with resummation. This result is in good agreement with the ATLAS UPC data [36]. In particular, the perturbative tail has been well described by the Sudakov formula as one would expect. In contrast to the acoplanarity of QCD dijets, the Sudakov resummation is not numerically important for the muon pair productions measured at ATLAS, since the combination of the single photon radiation contribution and the primordial contribution can explain the data well. Nevertheless, it is worth noting that the QED Sudakov resummation effects could be more important for other kinematic regions or electron pair productions.

We have also checked that the so-called nucleus dissociation contribution (or incoherent nucleon contribution) is negligible in this kinematics because of additional $1 / Z_{A}$ suppressions. This provides an important baseline for the central collisions, which we discuss in the following sections.
Medium effects in central collisions: Multiple scattering.-Both ATLAS and STAR focus on lepton pairs with small pair $P_{T}$, where the two photon scattering is the dominant channel in peripheral and even central heavy ion collisions [29,30,47]. The photon fluxes come from the charge distributions of incoming nuclei, and their contributions may not strongly depend on the centrality of the collisions. Therefore, in the following calculations, we assume that the total $P_{T}$ distribution from the incoming photons is the same for the peripheral and central collisions as in UPC events.

In non-UPC heavy ion collisions, the ATLAS and STAR data show that the lepton pair have accumulated additional $P_{T}$ broadening. This could be due to the interactions between the lepton pair and the medium. Because the leptons only carry electric charges, these interactions depend solely on the electromagnetic properties of the quark-gluon plasma created in these collisions.

The medium interactions are very much similar to the jet quenching and $P_{T}$ broadening mentioned in the introduction. Like the QCD case, the leptons suffer incoherent multiple scatterings with the medium. To evaluate this contribution, we can follow the $P_{T}$-broadening calculations in QCD [7,48]. The multiple photon exchanges between the lepton and the medium can be formulated in a QED type time-ordered Wilson line

$$
\begin{aligned}
\mathcal{U}_{\mathrm{QED}}\left(x_{\perp}\right)= & T \exp \left[-i e \int d z^{-} \int d^{2} z_{\perp} G\left(x_{\perp}-z_{\perp}\right)\right. \\
& \left.\times \rho_{e}\left(z^{-}, z_{\perp}\right)\right]
\end{aligned}
$$

where $\rho_{e}\left(z^{-}, z_{\perp}\right)$ is the electric charge source of the medium. The photon propagator $G\left(x_{\perp}\right)$ is defined as

$$
\begin{aligned}
G\left(x_{\perp}\right) & =\frac{1}{(2 \pi)^{2}} \int d^{2} q_{\perp} \frac{1}{q_{\perp}^{2}+\lambda^{2}} e^{i q_{\perp} \cdot x_{\perp}} \\
& =\frac{1}{2 \pi} K_{0}\left(\lambda x_{\perp}\right),
\end{aligned}
$$

where $\lambda$ acts as an IR regulator similar to the Debye mass in QED. Analogous to the QCD $q \bar{q}$ dipole calculation, the QED multiple scattering amplitude between the QED leptonic dipole with size $r_{\perp}$ and target medium can be written as

$$
\left\langle\mathcal{U}_{\mathrm{QED}}\left(b_{\perp}+\frac{1}{2} r_{\perp}\right) \mathcal{U}_{\mathrm{QED}}^{\dagger}\left(b_{\perp}-\frac{1}{2} r_{\perp}\right)\right\rangle=\exp \left[-\frac{Q_{s e}^{2} r_{\perp}^{2}}{4}\right]
$$

where the analog of saturation momentum in QED $Q_{s e}^{2} \equiv\left(e^{4} / 4 \pi\right) \ln \left(1 / \lambda^{2} r_{\perp}^{2}\right) \int d z^{-} \mu_{e}^{2}\left(z^{-}\right)$. Here, $\mu_{e}^{2}$ is related to the local charge density fluctuations. The dipole size $r_{\perp}$ is large in the soft momentum transfer region, which makes 
$Q_{s e}^{2} r_{\perp}^{2} \sim 1$. Therefore, we need to take into account the multiple scattering effects.

If we compare the above dipole to the QCD dipole $[49,50]$, we will find the following differences. First, because the couplings in QED and QCD are dramatically different, this introduces a major difference for the medium $P_{T}$-broadening effects, in addition to the difference in the Sudakov effects mentioned above. Second, the saturation scales depend on the charge density. Since only quarks carry electric charge, the QED saturation scale will depend on the quark density, whereas the QCD saturation scale depends on both quark and gluon density. Their densities are proportional to the respective degree of freedoms if we assume the ratio of the thermal distributions of quarks and gluons: $\frac{21}{2} N_{f}: 16$ [51]. Here $N_{f}$ is the number of active flavors. After accounting for the electric charge and color factor differences in the multiple scattering, we estimate the ratio between the QED and QCD saturation scales as

$$
\frac{\left\langle\hat{q}_{\mathrm{QED}} L\right\rangle}{\left\langle\hat{q}_{\mathrm{QCD}} L\right\rangle}=\frac{\alpha_{e}^{2}}{\alpha_{s}^{2}} \frac{\frac{21}{2} N_{f} \frac{2}{9}}{\frac{21}{2} N_{f} \frac{2}{9}+16 \frac{1}{2}}=\frac{\alpha_{e}^{2}}{\alpha_{s}^{2}} \times \frac{7}{15},
$$

for $N_{f}=3$ which gives $\sum_{u, d, s} e_{q}^{2}=N_{f} \frac{2}{9}$ and for QCD quark jets. Here $\langle\hat{q} L\rangle$ represents the saturation scale in dipole formalisms. For gluon jets, a factor of $C_{A} / C_{F}$ should be multiplied to the denominator. A few comments are in order. First, we assume that quarks and gluons are thermalized at the same time, which may not be true [51]. Second, we did not take into account a few detailed effects from the medium property, such as the associated Debye masses for QED and QCD. In addition, for the QCD case, there are length dependent double logarithms [52]. Last but not least, the medium path length $L$ can be different between the QED and QCD cases, since the electron pair can be created outside the medium. If all these effects are taken into account, the above simple formula cannot apply. Nevertheless, the above equation can serve as a simple formula for a rough estimate.

If we assume the QED multiple scattering limit, we can modify the above $W\left(b_{\perp} ; r_{\perp}\right)$ of Eq. (2) as

$$
\mathcal{N}_{\gamma \gamma}\left(b_{\perp} ; r_{\perp}\right) e^{-S_{u}\left(Q, m_{\mu} ; r_{\perp}\right)} e^{-\left(\left\langle\hat{q}_{\mathrm{QED}} L\right\rangle r_{\perp}^{2} / 4\right)}
$$

where the last factor comes from the medium contribution to the dilepton $P_{T}$-broadening effects. In Fig. 3, we show these effects by imposing two different values of the $\hat{q} L$. Comparing these curves to the ATLAS measurements, we conclude that the effective $\left\langle\hat{q}_{\mathrm{QED}} L\right\rangle$ range from $(100 \mathrm{MeV})^{2}$ in most central collisions to $(50 \mathrm{MeV})^{2}$ in noncentral collisions. Using Eq. (9) together with $\alpha_{s}=0.2$, we can find $\left\langle\hat{q}_{\mathrm{QCD}} L\right\rangle \sim 16 \mathrm{GeV}^{2}$ for central $\mathrm{PbPb}$ collisions at the LHC, which is in agreement with Refs. [23,24]. We can also estimate the QED energy loss [48]. However, it

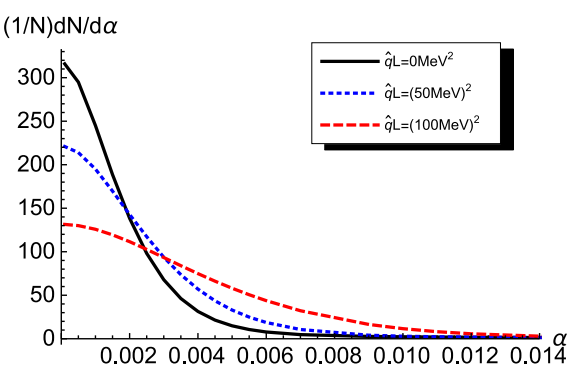

FIG. 3. Medium modifications to the acoplanarity distribution, with different values of the effective $\hat{q} L$.

is too small (few percent of the $P_{T}$-broadening value) to have any observational effects.

Medium effects: Magnetic fields.-There has been a suggestion that the $P_{T}$ broadening could come from the magnetic effects of the medium [30] as a result of the Lorentz force: $\vec{B} \times \vec{V}$, where $\vec{B}$ and $\vec{V}$ are the magnetic field vector and the lepton's velocity, respectively. The lepton bending is strongly correlated with the directions of the magnetic field and the lepton's momentum. If we can measure these correlations, we will be able to disentangle these mechanisms.

The initial magnetic fields generated by the colliding nuclei could contribute to an additional $P_{T}$-broadening effects. However, this effect is completely canceled out by the effects from the electric fields in the leading power of $q_{\perp} / P_{\perp}$ [37,53]. This cancellation is also consistent with a factorization argument that the final state interaction effects vanish in this process because of the opposite charges of the lepton pair.

Some theorists have suggested that there is a residual coherent magnetic field in the quark-gluon plasma after the collisions [54-56]. Because of the collision symmetry, the magnetic field only contains the perpendicular component $\vec{B}_{\perp}$. It has a nontrivial dependence on the impact parameter: increases from UPC to peripheral collisions but decreases toward more central collisions [54-56]. The ATLAS data do not appear to follow this trend.

This is very different from the incoherent multiple interaction effects discussed above, which increases monotonically with the centrality. Furthermore, because the Lorentz force vanishes along the direction of the magnetic field, the event plane dependence from the magnetic effects is expected to be quantitatively different with the one from the multiple scattering effects.

More importantly, the magnetic effects depend on the longitudinal velocity $v_{z}$ of the leptons. Therefore, if the lepton and the antilepton move in the same $z$ direction, the magnetic effects cancel out in the total pair $P_{T}$. Because of the linear dependence on $v_{z}$, the total $P_{T}$-broadening effects for the pair can be formulated as

$$
\left\langle\Delta p_{\perp}^{2}\right\rangle_{\mu^{+} \mu^{-}}^{B}=\left\langle\mathbf{P}_{\mathbf{m}}^{2}\left(b_{\perp}\right)\right\rangle\left[\tanh \left(y_{+}\right)-\tanh \left(y_{-}\right)\right]^{2},
$$




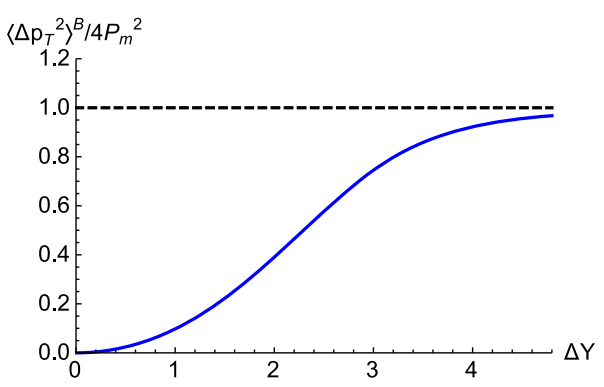

FIG. 4. Normalized magnetic effects on the $P_{T}$ broadening for the lepton pair as a function of their rapidity difference $\Delta Y=\left|y_{\mu^{+}}-y_{\mu^{-}}\right|$with $\left|y_{\mu}\right|<2.4$.

where $\left\langle\mathbf{P}_{\mathbf{m}}^{\mathbf{2}}\left(b_{\perp}\right)\right\rangle$ represents the average $P_{T}$ broadening depending on the centrality of the collisions, $y_{+}$and $y_{-}$are rapidities for the lepton and the antilepton, respectively. Figure 4 shows the normalized contribution as a function $\Delta Y=\left|y_{\mu^{+}}-y_{\mu^{-}}\right|$for a typical lepton transverse momentum $P_{\perp}=6 \mathrm{GeV}$. As expected, the magnetic effects on the $P_{T}$ broadening increases with $\Delta Y$. On the other hand, the multiple scattering effects discussed in the last section depend on the charge density of the medium and will not change in this rapidity range. Therefore, the difference between the $P_{T}$-broadening effects at different $\Delta Y$ can be used as an effective measure to the magnetic effects:

$$
\left[\left\langle\Delta p_{\perp}^{2}\right\rangle_{\Delta Y=3}-\left\langle\Delta p_{\perp}^{2}\right\rangle_{\Delta Y=0}\right]_{b_{\perp}} \propto\left\langle\vec{B}_{\perp}^{2}\right\rangle_{b_{\perp}}
$$

which depends on the centrality of heavy ion collisions. This will help to investigate other magnetic effects in heavy ion collisions, such as the chiral magnetic effects [54,56].

Summary and discussions. - We have investigated the dilepton production at very low total transverse momentum in heavy ion collisions to probe the electromagnetic property of the quark-gluon plasma. In the theoretical calculations, we take into account two important contributions: one is the soft photon radiation with Sudakov resummation; the other is the medium interaction leading to $P_{T}$ broadening for the leptons when they traverse the medium. By including the Sudakov resummation, we have shown that the theory predictions can well describe the azimuthal angular correlation of the lepton pair in the UPC events.

We have also shown that the $P_{T}$-broadening effects found by the ATLAS collaboration can be described by the multiple scattering of the leptons in the medium, where the effective $\left\langle\hat{q}_{\mathrm{QED}} L\right\rangle$ of order of $(50 \mathrm{MeV})^{2}$ to $(100 \mathrm{MeV})^{2}$ are in agreement with a parametric estimate of the QED and QCD effects of the quark-gluon plasma. We have investigated the $P_{T}$-broadening effects from the magnetic fields as well and pointed out there are a number of ways to distinguish these two mechanisms through a detailed study on (i) the centrality dependence of the effects, (ii) the correlation with the magnetic field (or reaction plane), and (iii) the rapidity dependence of the lepton pair. We emphasized that the magnetic effects depend on the rapidity difference between the lepton and the antilepton. This dependence can be used to determine the strength of the magnetic field.

In summary, our study demonstrated that the azimuthal correlation of the lepton pair in the low total transverse momentum region is a powerful tool to investigate the electromagnetic property of the quark-gluon plasma in heavy ion collisions. This shall stimulate further experimental and theoretical studies. In particular, we hope the rapidity dependence of the $P_{T}$-broadening effects can be measured to uniquely probe the magnetic effects.

We are grateful to Aaron Angerami, Brian Cole, Peter Jacobs, Volker Koch, Derek Teaney, and Zhangbu Xu for interesting discussions and comments. This material is based upon work partially supported by the Natural Science Foundation of China (NSFC) under Grant No. 11575070 and the U.S. Department of Energy, Office of Science, Office of Nuclear Physics, under Contract No. DE-AC02$05 \mathrm{CH} 11231$.

[1] K. Adcox et al. (PHENIX Collaboration), Phys. Rev. Lett. 88, 022301 (2001).

[2] C. Adler et al. (STAR Collaboration), Phys. Rev. Lett. 89, 202301 (2002).

[3] G. Aad et al. (ATLAS Collaboration), Phys. Rev. Lett. 105, 252303 (2010).

[4] S. Chatrchyan et al. (CMS Collaboration), Phys. Rev. C 84, 024906 (2011).

[5] M. Gyulassy and X.n. Wang, Nucl. Phys. B420, 583 (1994).

[6] R. Baier, Y. L. Dokshitzer, A. H. Mueller, S. Peigne, and D. Schiff, Nucl. Phys. B483, 291 (1997).

[7] R. Baier, Y. L. Dokshitzer, A. H. Mueller, S. Peigne, and D. Schiff, Nucl. Phys. B484, 265 (1997).

[8] R. Baier, Y. L. Dokshitzer, A. H. Mueller, and D. Schiff, Nucl. Phys. B531, 403 (1998).

[9] B. G. Zakharov, JETP Lett. 63, 952 (1996); 65, 615 (1997).

[10] M. Gyulassy, P. Levai, and I. Vitev, Nucl. Phys. B571, 197 (2000).

[11] U. A. Wiedemann, Nucl. Phys. B588, 303 (2000).

[12] X. N. Wang and X. f. Guo, Nucl. Phys. A696, 788 (2001).

[13] P. B. Arnold, G. D. Moore, and L. G. Yaffe, J. High Energy Phys. 06 (2002) 030.

[14] J. Casalderrey-Solana, J. G. Milhano, and U. A. Wiedemann, J. Phys. G 38, 035006 (2011).

[15] J. Casalderrey-Solana, D. C. Gulhan, J. G. Milhano, D. Pablos, and K. Rajagopal, J. High Energy Phys. 10 (2014) 019; 09 (2015) 175.

[16] K. M. Burke et al. (JET Collaboration), Phys. Rev. C 90, 014909 (2014).

[17] G. Y. Qin and B. Muller, Phys. Rev. Lett. 106, 162302 (2011); 108, 189904(E) (2012).

[18] C. Young, B. Schenke, S. Jeon, and C. Gale, Phys. Rev. C 84, 024907 (2011). 
[19] W. Dai, I. Vitev, and B. W. Zhang, Phys. Rev. Lett. 110, 142001 (2013).

[20] X. N. Wang and Y. Zhu, Phys. Rev. Lett. 111, 062301 (2013).

[21] A. H. Mueller, B. Wu, B. W. Xiao, and F. Yuan, Phys. Lett. B 763, 208 (2016).

[22] A. H. Mueller, B. Wu, B. W. Xiao, and F. Yuan, Phys. Rev. D 95, 034007 (2017).

[23] L. Chen, G. Y. Qin, S. Y. Wei, B. W. Xiao, and H. Z. Zhang, Phys. Lett. B 773, 672 (2017).

[24] L. Chen, G. Y. Qin, S. Y. Wei, B. W. Xiao, and H. Z. Zhang, Phys. Lett. B 782, 773 (2018).

[25] L. Chen, G. Y. Qin, L. Wang, S. Y. Wei, B. W. Xiao, H. Z. Zhang, and Y. Q. Zhang, Nucl. Phys. B933, 306 (2018).

[26] M. J. Tannenbaum, Phys. Lett. B 771, 553 (2017).

[27] L. Adamczyk et al. (STAR Collaboration), Phys. Rev. C 96, 024905 (2017).

[28] M. Gyulassy, P. Levai, J. Liao, S. Shi, F. Yuan, and X. N. Wang, Nucl. Phys. A982, 627 (2019).

[29] M. Aaboud et al. (ATLAS Collaboration), Phys. Rev. Lett. 121, 212301 (2018).

[30] J. Adam et al. (STAR Collaboration), Phys. Rev. Lett. 121, 132301 (2018).

[31] C. A. Bertulani and G. Baur, Phys. Rep. 163, 299 (1988).

[32] G. Baur, K. Hencken, and D. Trautmann, Phys. Rep. 453, 1 (2007).

[33] J. Adams et al. (STAR Collaboration), Phys. Rev. C 70, 031902 (2004).

[34] C. A. Bertulani, S. R. Klein, and J. Nystrand, Annu. Rev. Nucl. Part. Sci. 55, 271 (2005).

[35] A. J. Baltz et al., Phys. Rep. 458, 1 (2008).

[36] ATLAS Collaboration, CERN LHC, Report No. ATLASCONF-2016-025.

[37] S. Klein, A. Mueller, B. Xiao, and F. Yuan (to be published).
[38] S. R. Klein, J. Nystrand, J. Seger, Y. Gorbunov, and J. Butterworth, Comput. Phys. Commun. 212, 258 (2017).

[39] S. R. Klein, Phys. Rev. C 97, 054903 (2018).

[40] W. Zha, L. Ruan, Z. Tang, Z. Xu, and S. Yang, Phys. Lett. B 781, 182 (2018).

[41] S. R. Klein and J. Nystrand, Phys. Rev. C 60, 014903 (1999).

[42] A. J. Baltz, Y. Gorbunov, S. R. Klein, and J. Nystrand, Phys. Rev. C 80, 044902 (2009).

[43] P. Sun, C.-P. Yuan, and F. Yuan, Phys. Rev. D 92, 094007 (2015).

[44] J. C. Collins and D. E. Soper, Nucl. Phys. B193, 381 (1981); B213, 545 (1983).

[45] J. C. Collins and D. E. Soper, Nucl. Phys. B194, 445 (1982).

[46] J. C. Collins and D. E. Soper, Nucl. Phys. B197, 446 (1982).

[47] J. Adam et al. (ALICE Collaboration), Phys. Rev. Lett. 116, 222301 (2016).

[48] R. Baier, Y. L. Dokshitzer, A. H. Mueller, S. Peigne, and D. Schiff, Nucl. Phys. B478, 577 (1996).

[49] E. Iancu, A. Leonidov, and L. McLerran, arXiv:hep-ph/ 0202270.

[50] A. H. Mueller, Nucl. Phys. B558, 285 (1999).

[51] J. P. Blaizot, B. Wu, and L. Yan, Nucl. Phys. A930, 139 (2014).

[52] T. Liou, A. H. Mueller, and B. Wu, Nucl. Phys. A916, 102 (2013).

[53] D. Ivanov and K. Melnikov, Phys. Rev. D 57, 4025 (1998).

[54] D. E. Kharzeev and H. J. Warringa, Phys. Rev. D 80, 034028 (2009).

[55] M. Asakawa, A. Majumder, and B. Muller, Phys. Rev. C 81, 064912 (2010).

[56] V. Koch, S. Schlichting, V. Skokov, P. Sorensen, J. Thomas, S. Voloshin, G. Wang, and H. U. Yee, Chin. Phys. C 41, 072001 (2017), and references therein. 